KARL LÖWITH

MeIN LebEN IN DeUTSChLAND 


\author{
Karl Löwith
}

\title{
Mein Leben in Deutschland vor und nach 1933
}

\author{
EIN BERICHT
}

Mit einem Vorwort von Reinhart Koselleck und einer Nachbemerkung von Ada Löwith

J.B. Metzlersche Verlagsbuchhandlung

Stuttgart 
CIP-Kurztitelaufnahme der Deutschen Bibliothek

Löwith, Karl:

Mein Leben in Deutschland vor und nach 1933:

e. Bericht/Karl Löwith

Mit e. Vorw. von Reinhart Koselleck

u. e. Nachbemerkung von Ada Löwith. -

Stuttgart: Metzler, 1986.

ISBN 978-3-476-00590-8

ISBN 978-3-476-03222-5 (eBook)

DOI 10.1007/978-3-476-03222-5

(C) Springer-Verlag GmbH Deutschland 1986

Ursprünglich erschienen bei J. B. Metzlersche

Verlagsbuchhandlung und Carl Ernst Poeschel

Verlag GmbH in Stuttgart 1986 


\section{INHALT}

Vorwort von Reinhart Koselleck IX

Einleitung XVI

$$
1914-1933
$$

Krieg und Gefangenschaft 1

Nietzsche vor und nach Hitler 4

Österreicher, Deutsche und Italiener 6

Der Empfang in der Heimat 8

Der "Frontparagraph" 9

Nach dem Krieg 13

Zwei deutsche Männer 16

Meine erste Freundschaft nach dem Krieg 18

Der Kreis um Stefan George und die Ideologie des Nationalsozialismus

19

Oswald Spengler und Karl Barth 24

In Freiburg bei Edmund Husserl 26

Martin Heideggers Philosophie der Zeit (1919-1936) 27

Heideggers Übersetzung des »je eigenen Daseins« in das »deutsche Dasein« 32

Heideggers Persönlichkeit 42

Der umgekehrte Aufbruch Dr. B.'s 45

Der Geist und das Christentum sind in Deutschland ein Anachronismus 49

B.'s Stellung zur Judenfrage 54

Mein letztes Wiedersehen mit Husserl in Freiburg 1933 und mit Heidegger in Rom $1936 \quad 56$

Meine Freunde aus der Freiburger Studienzeit $\quad 59$

Die Auszehrung alles Bestehenden durch die Inflation $\quad 60$

Als Hauslehrer in Mecklenburg

61

Die Flucht aus der Zeit nach Italien

62

Rückkehr nach Marburg und Habilitation 64

70. Geburtstag und Tod meines Vaters 66 
Inhalt

Drei Vorzeichen des Umsturzes

Vor dem Umsturz 71

Die deutsche "Erhebung" von 1933 und meine letzte Marburger Vorlesung $\quad 74$

\section{$1934-1936$}

Mein Abschied von Marburg $\quad 81$

Italiener und Deutsche 82

Nationalsozialistische Professoren in Rom

Zwei deutsche Institutsdirektoren $\quad 87$

Die deutschen Emigranten in Rom 89

Russische Emigranten in Italien und Japan 94

Die Vertreibung der Juden aus Italien 95

Eine japanische und eine deutsche Naivität $\quad 96$

Jüdische und arische Schicksale der Marburger Universität $\quad 99$

Der Reflex der deutschen Ereignisse in Italien 101

Auf dem Prager Philosophiekongreß (1934) 103

Die Entziehung des Lehrauftrags und meine

Reise nach Deutschland 104

Rückkehr über Paris nach Italien 105

Im Flugzeug nach Istanbul 107

Berufung nach Japan und Abschied von

Deutschland und Europa 108

Verlegermiseren $\quad 109$

$$
1936-1939
$$

Ankunft in Japan 111

Ein englischer Kollege 111

Eine italienische Exzellenz und ein deutscher Geheimrat in Sendai 112

Die deutschen Emigranten in Japan 115

Nationalsozialismus in Karuizawa 117

Mein Verkehr mit Deutschen in Japan 119

Die deutschen Ereignisse von 1936-39 125 
Inhalt

Zwei arische Emigranten

127

Wie für mich die Trennung von Deutschen und Juden begann

131

Deutscher und Jude zugleich

131

Die deutsche Vereinfachung und der deutsche Protest

132

Nachwort 136

Noch ein Nachwort

137

Anmerkungen $\quad 140$

Curriculum vitae (1959) 146

Nachbemerkung von Ada Löwith 158 


\section{VORWORT}

Karl Löwiths Lebensbericht aus dem Jahre 1940 wurde durch einen äußeren Anlaß hervorgerufen. Von der Harvard-University ging ein Preisausschreiben aus, um Erfahrungsberichte von Augenzeugen aus Deutschland vor und nach $1933 \mathrm{zu}$ sammeln. Über den Verlauf des Verfahrens und den Verbleib der Einsendungen konnte bisher nichts ermittelt werden. Jedenfalls erhielt Löwith keinen Preis, und das verwundert kaum, denn "philosophische Erwägungen über die Vergangenheit « waren nicht erwünscht. Nun hat Löwith zwar weder eine philosophische Autobiographie verfaßt, noch hat er sich in allgemeinen kulturkritischen Betrachtungen ergangen, aber der Bericht fließt natürlich aus seiner unverwechselbaren Handschrift. Es ist die Handschrift des Philosophen, der ein biologisches Studium zurückgelegt hatte. Die Nüchternheit und Prägnanz einer mikroskopischen Sicht wird mit der Unmittelbarkeit und Klarheit phänomenologischer Beschreibung verbunden.

Es handelt sich also nicht um Memoiren im Rückblick, wie sie heute erscheinen und die aus der Vergangenheit zu retten suchen, was möglich - oder unmöglich ist. Eher handelt es sich um eine Zwischenbilanz, niedergeschrieben im japanischen Exil, um ein Innehalten, das noch von der unmittelbaren Betroffenheit zeugt, aus der sich Löwith mit der unerbittlichen Konsequenz seines Denkens zu befreien sucht. Es ist ein dramatisches Dokument, das nicht bis ins Letzte hinein kunstvoll komponiert ist, sondern mit tagebuchartiger Spontaneität immer von neuem einsetzt, das viele Briefe sprechen läßt und dem gedruckte Beigaben aus der nationalsozialistischen Selbstherrlichkeit eingefügt sind, die Löwith mit wacher Neugierde, gedämpftem Zorn und steigender Verachtung gesammelt hat. Es ist ein Dokument, dem sich die Spuren unvermittelbarer Erfahrung eingeschrieben haben. Darin liegt seine nicht überholbare Gegenwärtigkeit.

Die Gliederung ist - wechselweise - chronologisch und sachlich. Damit wird der doppelte Zugriff deutlich, werden zwei Ebenen umrissen: einerseits werden die persönlichen Erlebnisse berichtet, die auf die Wende von 1933 voraus- oder zurückweisen, zum anderen werden die Erfahrungen reflektiert, die Herausforderungen der Zeitgeschichte, soweit sie in das Leben des Autors eingegriffen und ihn zu einer Antwort genötigt haben. 
Die Biographie beginnt scheinbar normal, mit der Jugend eines Jugendbewegten, der aus einem gut bürgerlichen Künstlerhaus stammt. Der Vater war ein konfessionslos gewordener Jude aus Mähren, dessen Vaterland Deutschland und dessen Heimat München wurde, wo er als Maler eine hoch geachtete Stellung gewonnen hatte. Es folgen die freiwillige Meldung zum Kriegsdienst, Frontkampf im Regiment des Ritters von Epp, schwere Verwundung und Gefangenschaft in Italien, Rückkehr nach München, Begegnung mit Max Weber, das Studium bei Husserl und Heidegger, Habilitation in Marburg und erfolgreiche Dozententägigkeit - bis zum Jahre 1933. Insoweit lesen wir eine gelungene, wenn auch im Rahmen des Bildungsbürgertums typische Biographie.

1933 erfolgt die Ächtung als Jude, nicht völlig überraschend, aber alle Fasern der bürgerlichen Existenz zerschneidend, - scheinbar verzögert infolge der Kriegsteilnahme, - in Wirklichkeit noch demütigender, als ob die Kriegsteilnahme, eine wissenschaftliche Qualifikation darstelle, die Löwith als Jude abgesprochen wurde. Seit 1933 werden Löwith Alternativen aufgenötigt, die er sich nicht gesucht hat: Jude sein zu sollen, sein Amt aufgeben zu müssen, nach Italien zu entweichen, als Exilierter und nicht als Emigrant. Nach dem Auslaufen eines Rockefeller-Stipendiums die Suche nach einer Stellung in der restlich verbliebenen Welt. Schließlich, kurz vor der erneuten Vertreibung aufgrund der italienischen Rassengesetze, die rechtzeitige Übersiedlung nach Sendai, wo ihm Schüler und Freunde allem Einspruch deutscher Dienststellen zum Trotz eine Professur verschafft hatten. Der Bericht endet vor der letzten, ebenfalls gerade noch rechtzeitigen Flucht in die USA, wohin Löwith und seine Frau kurz vor Pearl Harbour entkommen konnten.

Dieser äußere Lebenslauf eines Gejagten, der von seiner Natur auf Kontemplation eingestimmt war, ist schon aufreibend genug, um den Leser anzusprechen und zu beanspruchen. Aber die wirkliche Herausforderung liegt in den Reflexionen, die Löwith beiläufig notiert. Sie lassen sich nicht mehr zusammenfassen, weil sie den einmaligen Situationen verhaftet bleiben, denen sie entsprangen.

Die biographischen Etappen werden zumeist durch Personen markiert, mit denen Löwith zusammentraf, sich fand oder auseinandersetzen mußte. Er ist ein Meister des Kurzporträts, psychologisch und physiognomisch, gewürzt durch Anekdoten, situativ nicht mehr auflösbare Dialoge und durch lakonische Kommentare, deren Knapp- 
heit nicht zu unterbieten ist. Löwith schreibt einen taciteischen Stil. Hier finden sich Charakteristiken von Max Weber und Albert Schweitzer, von Bultmann oder von Carl Schmitt, um nur vier Extreme zu nennen, mit denen Löwith nicht zufällig zusammengetroffen ist. Hier findet sich vor allem die autobiographische Genese der Heidegger-Kritik, die später, - Denker in dürftiger Zeit 1953 erschienen ist. Die eindeutige Anerkennung und die entschiedene Distanzierung von seinem Lehrer Heidegger bündeln sich zu einem Rätsel, das weder bloß psychologisch noch bloß soziologisch noch bloß philosophisch gelöst werden kann, weil immer ein Winkel aus der jeweils anderen Perspektive uneinsichtig bleibt. Löwith leuchtet in jeden Winkel, redlich im Dank, aber vom Dank unbeirrt.

Der persönlich einmalige Bericht gewinnt schließlich exemplarischen Rang, sobald die Ereignisfolgen vor und nach der Machtergreifung Hitlers geschildert werden, kraft seines ungetrübten Blicks, den sich Löwith gewahrt hat. Die politische Semantik verrät noch die zeitliche Nähe und Verstrickung, die - vergangene - Gegenwärtigkeit des schleichenden oder offenen Terrors, dem Löwith mit kühler Ruhe begegnet, auch wenn gelegentlich ein provozierter $\mathrm{Haß}$ aufbricht, minimaler Schutz vor dem Zugriff der nationalsozialistischen Deutschen.

Die Etappen des Löwith aufgenötigten Lebensweges zeigen per negationem die Geschichte der deutschen Misere, der Lächerlichkeit, des Ehrgeizes, des Opportunismus und der Anpassung, der Feigheit und des Fanatismus, die sich allesamt gegenseitig abstützen und hochtreiben. Der nationalsozialistische Einparteienstaat mit seinen Nischen und Absurditäten und mit seinen wohlorganisierten Verbrechen erweist sich zugleich als Voraussetzung und als Ergebnis von Verhaltensweisen, die Löwith - für uns Deutsche peinlich - und exakt registriert. Meistens erübrigt die Beobachtung jeden Kommentar, gelegentlich wird sie mit der Feder des großen Moralisten konturiert. Die seltenen Fälle von Mut und Zivilcourage werden sorgsam festgehalten, aber auch sie machen deutlich, wo sie auflaufen oder leerlaufen, wie sie erstickt und in die Privatheit abgedrängt werden.

Was sich der normalen Einbildungskraft entzieht, was seit 1933 überhaupt möglich wurde, das bezeugen die Stationen eines - ex post gesehen vergleichsweise glimpflich verlaufenen - Lebens, von denen Löwith berichtet, weil sie ihn und seine Frau betroffen haben: die Judengesetze, "die politische Zoologie der Rassenprozente" machten 
aus seiner protestantisch getraute Ehe eine "Mischehe" -, die obendrein willkürliche Anwendung der Judengesetze, der Entzug von Gehalt und Kriegsrente - bei gleichzeitiger Aushändigung einer Kriegsmedaille in Rom -, die Sperrung des Vermögens, die Schließung der Hotels für Juden, die Bespitzelung und Denunziation, der Zwang zum Verschweigen der Wahrheit, die Sperre der Verlage, das Verbot der Bücher, also ein Zwang zum Verstummen, fast schon ein Verlust der deutschen Sprache als eines öffentlichen Kommunikationsmittels, all diese Stationen, so lächerlich und banal wie furchtbar und lähmend, werden minutiös dargestellt. Dabei lag die systematische Vernichtung der Juden noch jenseits des Berichtszeitraumes, was der Leser nicht vergessen darf, auch wenn die Konzentrationslager zum selbstverständlichen Wissen gehörten.

Die verlorene Heimat verstummt, weil die Zensur nicht mehr durchläßt, was erfragt werden könnte. Aber der politisch bewußt vollzogene Abschied kann nicht gelingen, weil sich der drohende Schatten des nationalsozialistischen Regimes auch in Italien und Japan zunehmend auf die Exilierten senkt. Doch Löwith verliert kein Wort der Angst. So wurde Löwith, wie gesagt, von einer Alternative in die andere gedrängt, ohne daß er sie sich je gesucht hätte.

Zwei große Themen sind es, die er - entlang allen Einzelgeschichten - immer wieder reflektiert: Der Verfall der deutschen bürgerlichen Welt und die ihm aufgenötigte Spaltung seiner Existenz in die eines Deutschen und eines Juden. Beide Themen hängen unmittelbar zusammen. Sie prägen den ganzen Bericht.

Das Jahr 1933 wird als tiefer Einschnitt, als Einbruch erfahren. Und das nicht nur wegen seiner persönlichen Diskriminierung als "Jude", sondern ebenso als Ergebnis einer langen Periode der Dekadenz des Bürgertums, das durch Krieg und Inflation gebeutelt, einer Selbstzerstörung anheimfiel, aus der es keinen Ausweg zu geben schien. Derartige Zeugnisse können heute kaum unterschätzt werden. Denn Löwith läßt keinen Zweifel daran, daß er, der sich vor 1933 als unpolitisch betrachtet und auch so gelebt hatte, an der damals eingängigen Bildungskritik, an der intellektuellen Auflösung des Christentums und des Humanismus beteiligt war, und sei es auch nur als geschichtlich bewußter Philosoph, als konsequenter Analytiker dieses Prozesses. Aber daß der Weg nicht nur von Hegel über Marx zu Nietzsche führen sollte, sondern ebenso weiter zu Jacob Burckhardt, das war schon in seiner intellektuellen Biographie der zwanziger Jahre 
angelegt. Nur wurde die bewußt existentielle Wende erst vollzogen im Bannkreis des nationalsozialistischen Terrors, den Löwith als eine Konsequenz der deutschen Geschichte begreift.

Löwith bekennt sich ausdrücklich dazu, daß es keinen Weg zurück mehr geben könne, weder zurück zum Christentum, etwa aus Trotz gegen das neudeutsche Heidentum, noch zurück zum Judentum, aus dem er sich emanzipiert wußte, noch gar zurück zum klassischen Neuhumanismus, zu "Goethe«. Und Löwith hat es nie akzeptiert, sich zu der ihm aufgenötigten Alternative zu »bekennen«. Das wäre einer scheinbar freiwilligen, de facto erzwungenen politischen Option gleichgekommen, die ihn als Philosophen um seine geschichtlich reflektierte Identität gebracht hätte. So fand sich Löwith auf einen Weg gedrängt, auf dem allein er sich behaupten konnte, und zwar mit Würde, auch wenn er diesen Ausdruck als pathetisch abgewiesen hätte. Es war der Weg des philosophierenden Historikers in die konsequente Skepsis, und es war der Weg des geschichtlich reflektierenden Philosophen zu einer Sicht der Welt, die aller Geschichte vorausliegt.

So sah sich Löwith paradoxerweise genötigt, eine Tradition zu wahren, deren Fragwürdigkeit er durchschaut hatte, aber deren tatsächliches Gegenteil - und Ergebnis - die Barbarei war. Das Festhalten am nicht mehr Einholbaren trieb ihn aber nicht in die Verzweiflung, sondern stärkte seinen rigorosen $Z$ weifel, der sich allen Geschichten und allem Geschehen überlegen weiß. Es wäre ideologiekritisch anmaßend und philosophisch unzureichend, den einmal gewonnenen Standpunkt konsequenter Skepsis nur als sozial oder politisch-biographisch bedingt zu erklären. Das bezeugt der vorliegende Bericht; Löwiths Immunität gegen jede Phrase, die ihn selbst zu unterkühlter Ideologiekritik befähigt, hat seit dem Ersten Weltkrieg seinen Lebensweg geprägt. Seine Ablehnung jeder vorschnellen oder scheinbar endgültigen Lösung impliziert eine durchdachte politische Haltung, die zu aktivieren ihm in Deutschland nach 1933, zum jüdischen Feind abgestempelt, nicht mehr möglich war. Schließlich gewann er in und mit seiner Skepsis eine Position, die jede falsche Konzession zu machen verbietet.

Die geschichtlich bedingte Entstehung und die philosophisch genuine Überzeugungskraft jenes Skeptizismus, den Löwith später auf seinem Heidelberger Lehrstuhl vertreten wird, sie werden in diesem Lebensbericht einsichtig und nachvollziehbar. 
Seine Distanz erlaubte es ihm, auch jene Zwischentöne herauszuhören, die etwas zwischen nationaldeutschen und gar nationalsozialistischen Juden auftauchten, und die so lächerlich wirken wie jene Juden, die ihn zu antisemitischen Bemerkungen herausforderten: obwohl beidesmal die bittere Ausweglosigkeit der Situation eine Erklärung, aber nicht den Schein einer Entschuldigung boten. - Oder er weiß zu unterscheiden zwischen dem treuherzigen SS-Studenten, der sich zum Abschied in Marburg bei ihm bedankt, und jenen Gestalten, die ohne Parteinummer hochklettern, weil sie sich voreilig anpassen, - obwohl sich beide auf ein deutsches Schicksal beriefen, das zwar keine Erklärung aber den Schein einer Begründung lieferte.

Löwith bleibt skeptisch gegenüber allgemeinen Volkscharakteristiken, aber als Moralist, der er auf den Spuren der Franzosen und Nietzsches auch war, riskiert er brillante Gesamturteile, - etwa im Vergleich zwischen Italienern und Deutschen -, die aufgrund ihrer Einseitigkeit nur wahr sein können: wahr im Sinne der Moralistik, nicht der blanken Faktizität. So beklemmend der Bericht wirkt, es ist auch ein kritisches Vergnügen, ihn zu lesen.

Die Edition wurde von der Verlagsredaktion um einige, wenige, Sätze gekürzt, ohne Sinn oder Zusammenhang zu zerstören. Die Nennung von Namen mit Anfangsbuchstaben wurde beibehalten, wo sie Löwith verwendet hat. Diese Schreibweise gehört zur Signatur der Zeit, in die der Bericht fällt. Die zu Buchstaben verkürzten Namen gerinnen gleichsam zu Typen oder Rollenträgern, deren Vielzahl oder Varianten sich der Leser hochrechnen kann. Löwith wollte nicht denunzieren, sondern demonstrieren. Ausgeschrieben wurden nur die Namen bekannter Figuren der Zeitgeschichte, aus Politik und Wissenschaft bis hin zu Spranger, dessen Opposition und deren betulichtörichte Vorträge in Japan Löwith auf einen gemeinsamen Nenner zu bringen weiß. Schließlich werden die Freunde mit vollem Namen zitiert, die zu nennen Löwith sich nicht scheuen mußte.

Löwith hat offenbar nicht daran gedacht, seinen Bericht nach der Rückkehr zu veröffentlichen. Er bekennt sich im Text mit Burckhardt zum »Gesetz der Verjährung . . ., das nicht bloß nach Jahren, sondern nach der Größe des Risses seine Entscheide gäbe«. Das war geschrieben worden vor der Vernichtung der Juden, für die es im Sinne des Wortes keine Verjährung geben kann. Daß alles Vorher und Nachher der Geschichte in Anbetracht von Welt und Ewigkeit dahinschwinde, gehört zu den Antworten, die Löwith auch in seiner Vita 1959 


\section{Vorwort}

formuliert hat. Diese Vita, vorgetragen in der Heidelberger Akademie der Wissenschaften, ist hinzugefügt worden: Sie zeugt von der erstaunlichen Kontinuität einer philosophischen Grundeinstellung, die sich, durch Terror und Exil bekräftigt, beidem gewachsen und überlegen weiß. Das macht Löwiths Lebensbericht erinnerungswert und denkwürdig.

z. Z. New York, im März 1986

Reinhart Koselleck 


\section{EINLEITUNG}

Die Unterscheidung der Geschichte Europas durch ein "vor" und "nach» Christus beherrscht zwar in Deutschland noch den Kalender, aber nicht mehr die Geister. Die aus dem Weltkrieg hervorgegangenen Diktaturen erheben, wie einst die Französische Revolution, den Anspruch, die gesamte Geschichte neu zu datieren. Und in der Tat, es läßt sich nicht leugnen, daß alles anders ist, als es war. Die Tatsache dieser Veränderung wird in Deutschland niemand bestreiten: Hitlers Gefolgschaft und ihre zum Schweigen verurteilten Gegner stimmen in diesem Punkt überein. Es ist, wie mir vor kurzem jemand aus Deutschland schrieb, mit Vielem »einfach vorbeik.

Die folgenden Aufzeichnungen wollen einiges Material zur Veranschaulichung dieses "Umbruchs « geben. Sie beruhen ausschließlich auf der Erinnerung eigener Erlebnisse sowie auf Briefen und andern authentischen Dokumenten, welche ich seit 1933 in der ihnen zukommenden Unvollständigkeit und Zufälligkeit aufbewahrt habe. Gemessen an den offiziellen Berichten des Nürnberger Parteitags oder auch an den inoffiziellen über die Konzentrationslager, sind die Worte und Handlungen, die mich persönlich betrafen, so unbedeutend wie das Geschick eines deutschen Privatdozenten im Vergleich zu einer totalen und systematischen Umwälzung. In diesem Mangel an exzessiven Geschehnissen liegt der Vorzug der folgenden Aufzeichnungen: sie geben nicht mehr und nicht weniger als ein alltägliches Bild von dem, was im beschränkten Umkreis eines unpolitischen Einzelnen wirklich geschah. - Nur in einem können sie nicht mehr der Wahrheit gemäß sein, nämlich im Ton. Die Erinnerung hat die Macht, selbst das Bitterste zu verwandeln, und was einer im Abstand von sechs Jahren erzählt, ist in den Besitz seines Lebens übergegangen, der das ursprüngliche Leid über den erlittenen Verlust ernüchtert und übertönt. Andrerseits sind die Ereignisse doch noch lebhaft genug, um die beteiligt gewesenen Menschen in einer Weise charakterisieren zu können, welche zeigt, daß sie einen auch heute noch, mehr als man wünschen kann, angehen. Es lag mir fern, die Schärfe des Urteils zu mildern, welche durch diese Nähe bedingt ist. 